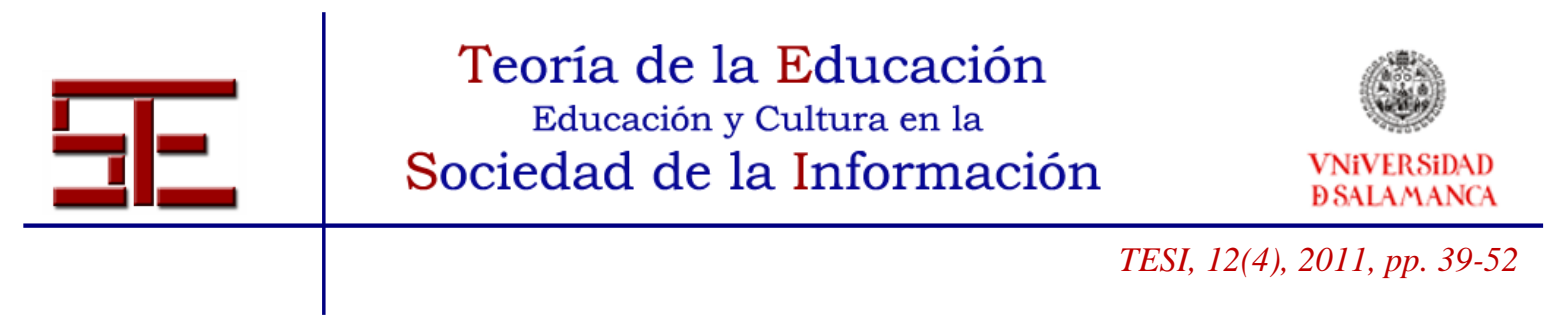

\title{
APRENDIZAJE COOPERATIVO Y HEURÍSTICO EN LA RED INTERNACIONAL E-CULTURAS
}

Resumen: Los entornos virtuales de aprendizaje, convertidos en plataformas digitales, favorecen la colaboración y la interacción entre usuarios, al mismo tiempo que permiten que la capacidad creativa se vea incrementada considerablemente. Sobre estas premisas se fundamenta el Programa Intercultural e-Culturas que se pone en marcha a través de la Red Internacional e-Culturas (http://www.e-culturas.org). Se trata de un proyecto colaborativo en red que tiene como fin hermanar a niños de diferentes nacionalidades para trabajar una serie de materiales interculturales. En la presente comunicación se exponen las líneas metodológicas básicas que sustentan el mismo.

Palabras clave: Aprendizaje colaborativo; entorno virtual aprendizaje; TIC.

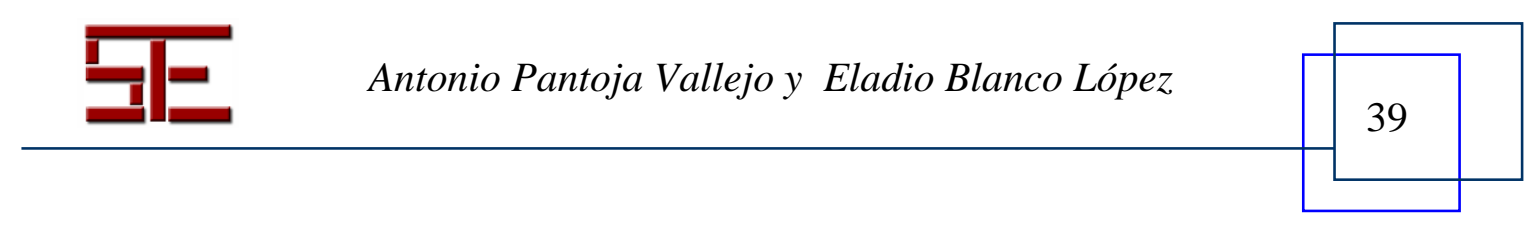




\title{
COOPERATIVE AND HEURISTIC LEARNING IN THE INTERNATIONAL NETWORK E-CULTURAS
}

\begin{abstract}
Virtual learning environments, converted to digital platforms, encourage collaboration and interaction between users, while allowing the creative capacity will be increased considerably. On this basis the Intercultural Program e-Culturas is based, being part of the International e-Culturas Network (http://www.e-culturas.org). This is a collaborative networking project that aims to twin children of different nationalities to work a series of cross-cultural materials. In the present paper is explained the basic methodology underlying it.
\end{abstract}

Keywords: Collaborative learning; virtual learning environment; ICT.

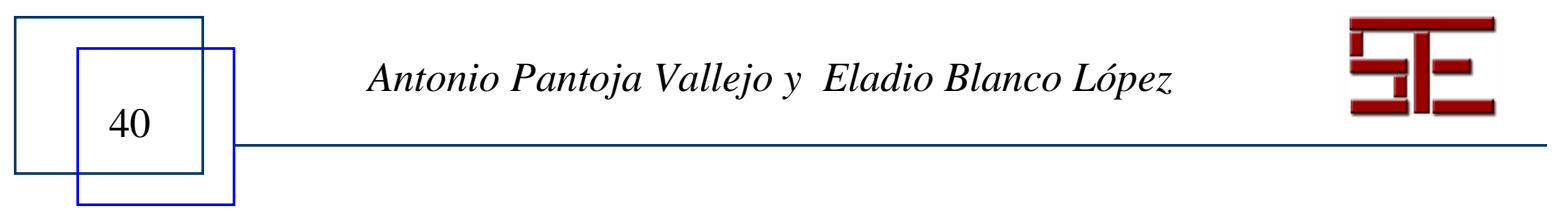




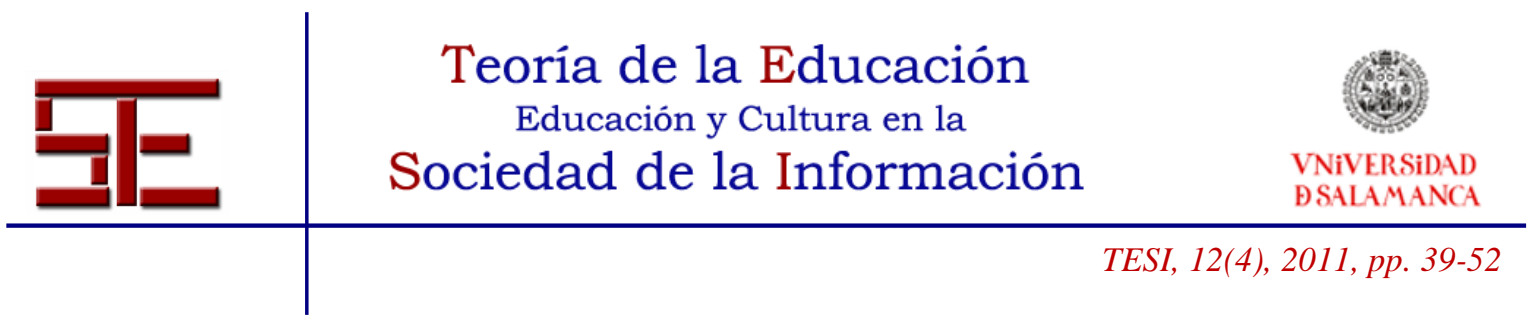

\title{
APRENDIZAJE COOPERATIVO Y HEURÍSTICO EN LA RED INTERNACIONAL E-CULTURAS
}

Fecha de recepción: 28/10/2011; fecha de aceptación: 25/11/2011; fecha de publicación: 20/12/2011

\author{
Antonio Pantoja Vallejo \\ apantoja@ujaen.es \\ Universidad de Jaén \\ Eladio Blanco López \\ elbloo@gmail.com \\ Universidad de Jaén
}

\section{1.- INTRODUCCIÓN}

Es un tópico redundar que en nuestra sociedad es cada vez más multicultural, las intensas migraciones en las últimas décadas han permitido que las sociedades ya no sean tan homogéneas y que configuren un tejido social más diverso y multicultural. Este proceso ha desencadenado un "reordenamiento" en el sistema social, político, económico y consecuentemente en el sistema educativo. Sumado a esto, el cambio vertiginoso de las sociedades actuales por el avance de las Tecnologías de Información y Comunicación (TIC) ha provocado que las fronteras de la comunicación se hagan menos distantes y más dinámicas, haciendo irremediable e imprescindible su uso en los contextos educativos.

Este escenario es el que ha originado el desarrollo, el diseño y la implementación del Programa intercultural e-Culturas desarrollado por el Grupo de Investigación IDEO y puesto en marcha mediante la "Red Internacional e-Culturas" (Pantoja et al., 2006; Pantoja et al., 2008). La idea se inscribe dentro de un convenio de colaboración entre la Universidad de Jaén (UJA) y distintas universidades de Latinoamérica, Portugal y Reino Unido. Se trata de un proyecto basado en el modelo constructivista y cognitivista de aprendizaje, que tiene por finalidad favorecer el encuentro y conocimiento cultural del alumnado de centros educativos españoles y latinoamericanos mediante el uso de TIC.

e-Culturas apuesta por una Educación Intercultural en el ámbito educativo, ya que la escuela constituye el primer espacio para la configuración de las relaciones sociales, en

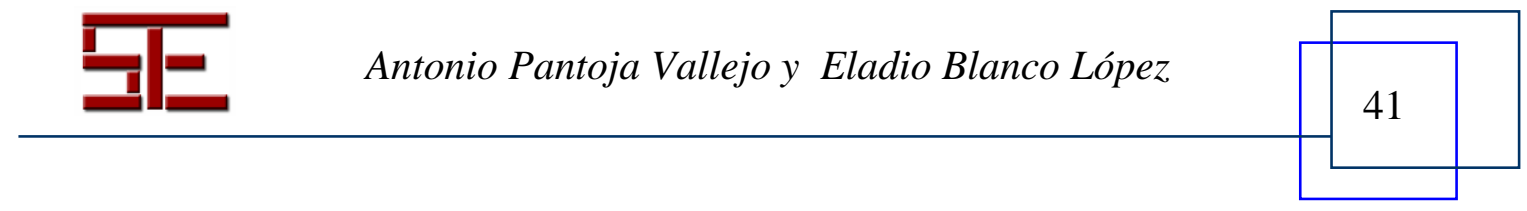




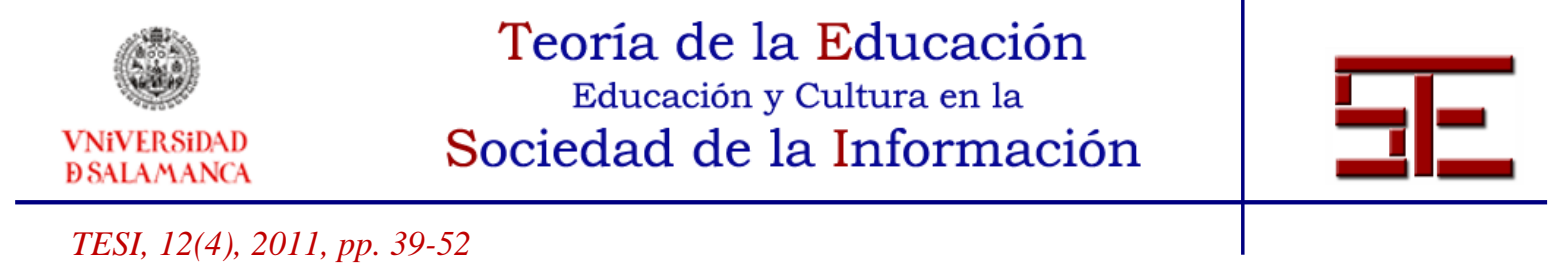

donde los alumnos establecen un marco de relaciones con los demás, empiezan a construir su identidad y percibir a los otros. Es en última instancia un escenario en donde se ponen a prueba los conflictos modernos en pro de la integración, la nueva y necesaria dimensión de la educación cívica y en cierta medida el pulso de la calidad de la democracia en la sociedad.

\section{2.- APRENDIZAJE COOPERATIVO Y HEURÍSTICO}

El aprendizaje cooperativo es un método docente que permite al alumnado el aprendizaje compartido, el enseñar y aprender en cooperación y los convierte en agentes activos de todo el proceso. Este tipo de aprendizaje favorece la instrucción compartida y el reparto de roles por parte de los propios alumnos integrados en grupos de trabajo. El profesor pasa a ser un coordinador y asesor. El aprendizaje cooperativo ofrece grandes posibilidades cuando se trabaja con ordenadores y el sistema informático favorece la interactividad. Entre sus ventajas destacan: construcción de nuevas ideas con la contribución de pares, lo cual favorece especialmente a los estudiantes que tienen más dificultades y enriquece a aquellos más aventajados; cada alumno puede aportar ideas y contribuir a la mejora del trabajo conjunto, lo que ayudará a los que tienen dificultades de aprendizaje; las actividades las propone el profesor y determina el rol de cada estudiante para la solución de este, por lo que cada alumno se responsabiliza de una parte de la solución de la tarea.

Por su parte, la metodología heurística orientada se adapta al aprendizaje creativo y facilita el descubrimiento personal del niño mediante el autoaprendizaje. Entre sus ventajas destacan: desarrolla estrategias personales, mantiene la motivación, permite dominar contenidos y descubrir principios. Con la metodología heurística el alumno busca y adquiere el conocimiento por sí mismo, de forma personal, creativa y no dirigida (Torre, 1991). La metodología heurística que se sigue posibilita un ambiente de trabajo, que facilita la capacidad de crear en el niño en su trabajo el ordenador, al mismo tiempo lo motiva para que aprenda por sí mismo y le ofrece pautas de trabajo cuando aparecen los conceptos curriculares.

\section{3.- FUNDAMENTACIÓN DE LA PROPUESTA}

Los centros educativos viven desde hace años una situación nueva como consecuencia del fenómeno multicultural, cada día más presente en las aulas. Ante esta realidad

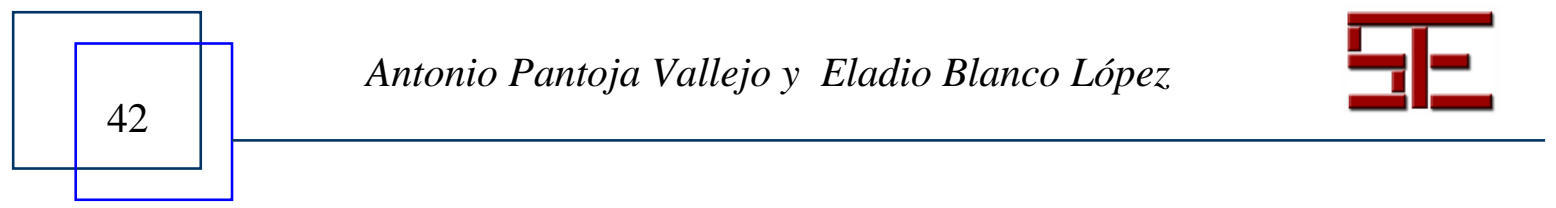




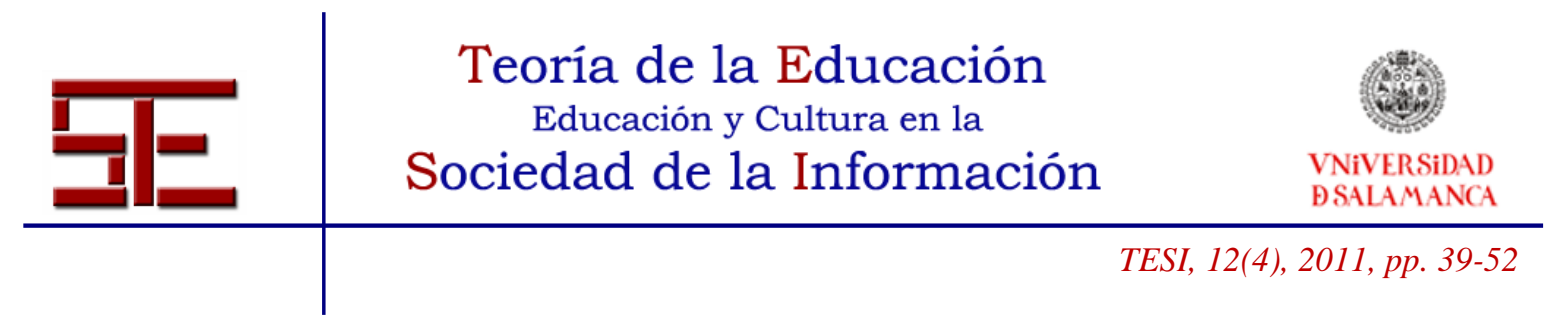

tenemos la necesidad imperiosa de dar respuesta a esta nueva situación, de manera que una vez detectadas estas necesidades hay que proceder a diseñar programas que den respuesta a una escuela cada día más multicultural, más abierta, dinámica y cambiante, desde el enfoque de educación intercultural que venimos defendiendo en este trabajo, basado en las nuevas tecnologías.

Los criterios que utilizamos para la fundamentación y el desarrollo del programa son los siguientes:

- Nuestro programa intenta favorecer, promover, facilitar el proceso de desarrollo de la identidad étnica-cultural de los alumnos, el pluralismo cultural y la educación para la ciudadanía. La reflexión y el análisis crítico personal es un componente esencial en el proceso de desarrollo de la identidad.

- La estructura jerárquica del programa responde a los modelos teóricos de desarrollo de la identidad étnico-cultural, que indican que es necesario comenzar por una reflexión personal sobre la propia identidad para después ser capaces de conocer al otro, para poder colocarse en "el lugar" del otro.

- Es un programa que se dirige a todo el alumnado: autóctono e inmigrante.

- La construcción positiva y la valoración de la identidad comporta un efecto positivo en la autoestima y en el autoconcepto del alumnado. El programa tiene una incidencia en el desarrollo de la dimensión afectiva.

- Se adopta el concepto de cultura como algo dinámico y no estático.

- El programa se implementa en el contexto natural del aula en donde se llevan a cabo procesos de aprendizaje.

- El programa utiliza módulos de contenido intercultural y como soporte las nuevas tecnologías de la información y la comunicación.

- El programa tiene en cuenta las finalidades educativas y unidades curriculares del centro.

- El Programa Intercultural e-Culturas tiene la "vocación" de contemplarse como un tema transversal dentro de otras áreas de orientación y contenido curricular, desde las áreas Social y Natural. Lo deseable por nuestra parte sería que, una vez aplicado el Programa Intercultural e-Culturas y llevada a cabo su valoración positiva se convirtieran sus contenidos en temas transversales en el currículum del alumno. Pero, obviamente, estas decisiones no nos corresponden. Sin embargo, sí hemos de tener muy en cuenta que un trabajo participativo con otros agentes nos permitan encontrar puente de conexión entre los contenidos del

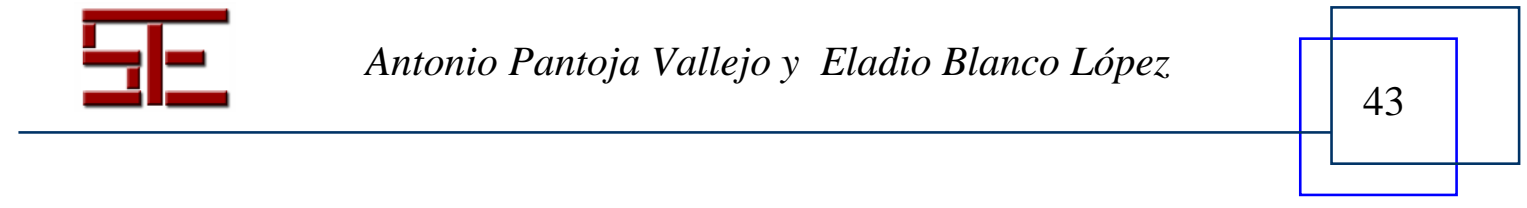




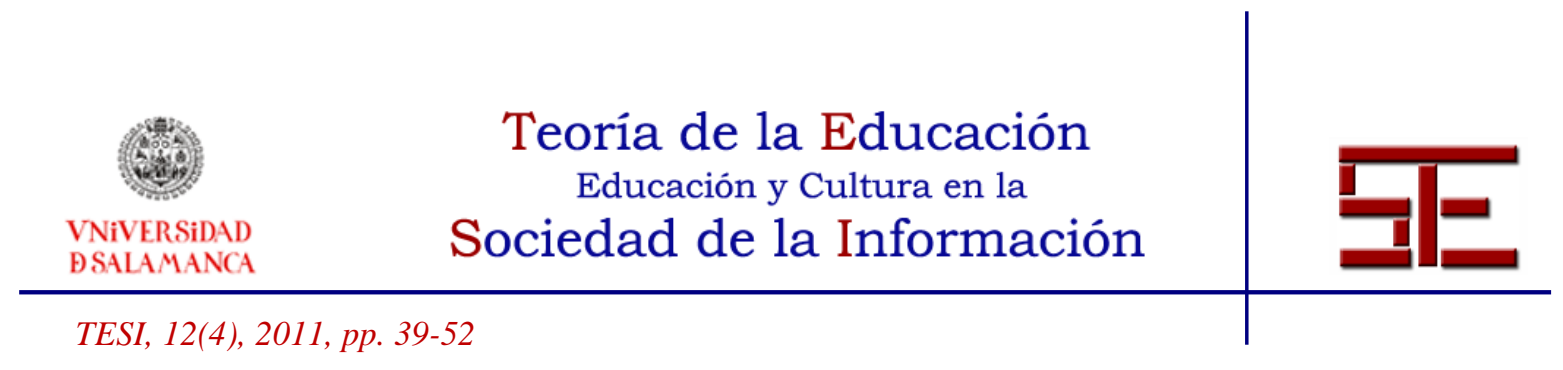

programa y los contenidos curriculares del alumno. Es decir, considerarse un eje transversal dentro de las demás áreas.

\section{1.- Metodología general del Programa}

La perspectiva metodológica del Programa se fundamenta en una concepción constructivista del aprendizaje, la utilidad del trabajo cooperativo, el contraste entre iguales, la necesidad de considerar la enseñanza como un proceso de investigación en la acción y los principios básicos del aprendizaje significativo. Todo esto nos lleva a establecer lo siguiente:

a) Para aprender algo los alumnos necesitan ver su utilidad, lo que supone la utilización de hechos, fenómenos y situaciones próximas.

b) La enseñanza es intencionada y mediatizada por los agentes (profesorado, familia, compañeros y aplicadora del programa), ya que por sí solo el alumno no descubre y aprende los conocimientos.

c) Las situaciones de aprendizaje deben favorecer el desarrollo personal, la cooperación, la creatividad, la crítica, la satisfacción por conocer y aprender, etc.

d) Plantear situaciones en que los alumnos identifiquen y reconozcan sus ideas, a partir de una reflexión, crítica e individual y de contraste con otros compañeros.

e) Implicar a los estudiantes en el proceso, de manera que tengan conciencia de que están trabajando con sus ideas iniciales a lo largo de la construcción del conocimiento, y que con la aplicación del programa se vaya modificando, sustituyendo o ampliando.

f) La metodología se basa en diversas técnicas para la consecución de los objetivos del programa.

Además, el programa busca desarrollar contextos de aprendizaje colaborativo en los que los estudiantes utilicen una variedad de recursos tecnológicos para apoyar el desarrollo de las actividades, transferencia de conocimiento, almacenamiento y extracción de la información; de allí que la modalidad de trabajo de este programa sea mayormente cooperativa y con uso de las TIC disponibles en los centros. Estas tecnologías a las que nos referimos son los ordenadores, Internet, CD/DVD, correo electrónico, cámara web, etc.

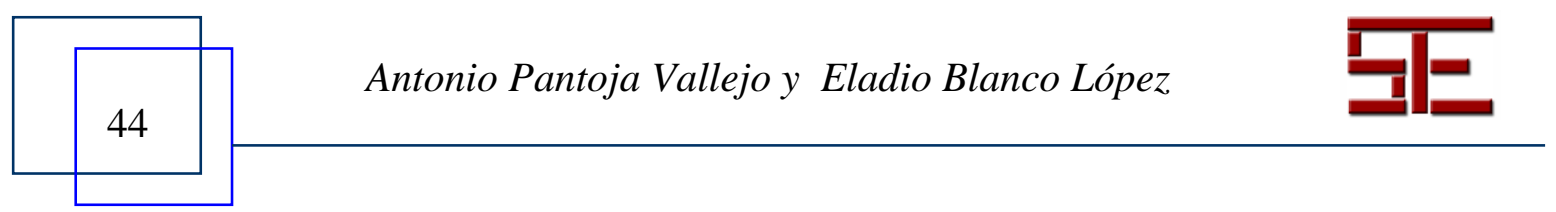




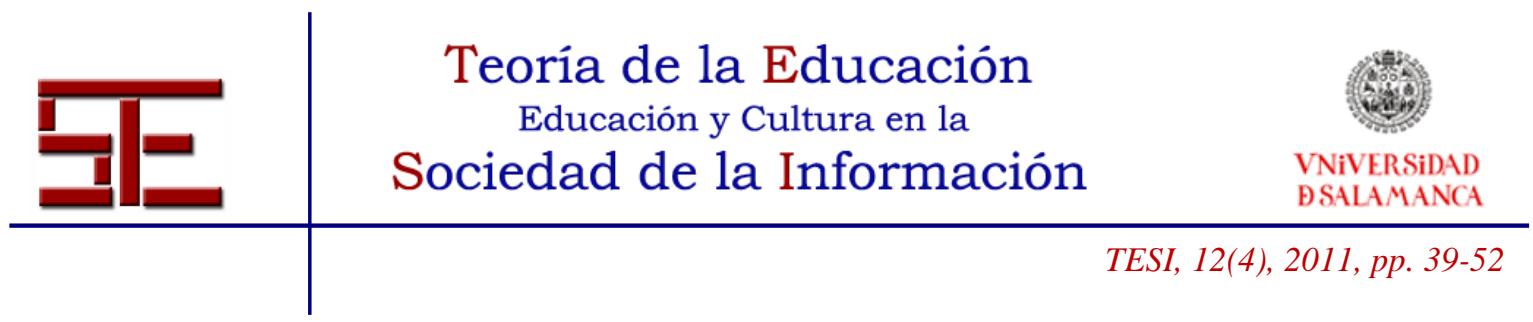

\section{2.- Fases en el desarrollo de cada módulo intercultural}

La propuesta metodológica es una adaptación de la desarrollada por Pantoja (1997), con los siguientes apartados:

a) PRESENTACIÓN: Es el resumen pormenorizado de los aspectos más importantes que presentan las actividades del módulo. La concreción de los contenidos que se desean conseguir.

b) OBJETIVOS: Todo aprendizaje debe tener una guía, un motivo que conseguir. Cada sesión de trabajo está basada en unos objetivos que resumen las capacidades a las que se debe llegar al finalizar el tiempo de interacción con la máquina.

c) PUESTA EN COMÚN: Es el punto de arranque de cada módulo. En él se comentan los proyectos que se propusieron en el módulo anterior, en su caso. $\mathrm{Su}$ duración es muy corta, aproximadamente 5 o 10 minutos.

d) DESCUBRE: Se introducen los nuevos conceptos de una forma heurística, inductiva, aprendizaje por descubrimiento, activa, creativa, lúdica, constructiva, socializada, cooperativa y colaborativa mediante el diálogo del grupo-aula y el trabajo en grupo pequeño, formado por los dos alumnos que trabajan en cada ordenador. Se da respuesta a los contenidos del módulo de una forma inductiva, proponiendo actividades ${ }^{1}$ que sean de fácil solución.

e) OBSERVA: Es un proceso de síntesis. En esta fase se explican y describen los conceptos introducidos en el apartado Descubre. Se revisa en grupo la teoría que se ha trabajado de forma inductiva y se comentan los principales aspectos teóricos que tienen que aprender los alumnos. Se intenta que nadie quede con dudas, intercambiando opiniones para comprobar si los alumnos han percibido aquello que se pretendía, de forma que no haya desfase entre lo que debían de haber deducido y lo que realmente han observado y retenido.

f) EXPERIMENTA: Conjunto de actividades individuales, aunque apoyadas en el intercambio de opiniones e información entre miembros de los equipos (niños hermanados) y dentro de las mismas aulas. En estas actividades el alumno integrará todo lo aprendido en el apartado Observa.

${ }^{1}$ En la página web de la Red internacional e-Culturas, dentro de cada módulo específico, existe una Oficina de Información con recursos concretos y enlaces a otras páginas web en los que el alumnado se podrá apoyar para la realización de las distintas actividades planteadas.

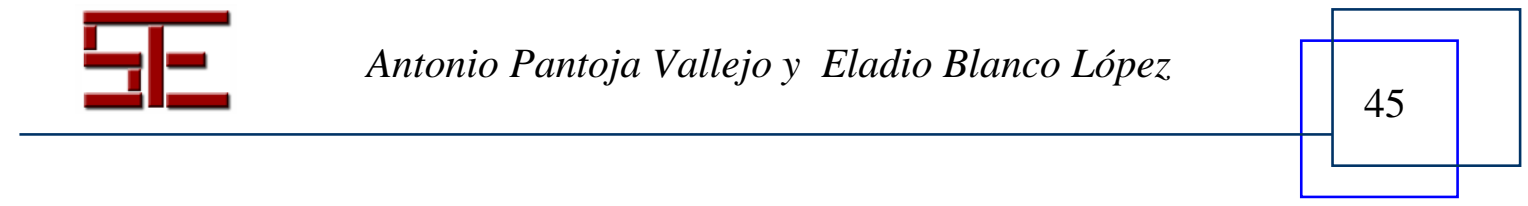




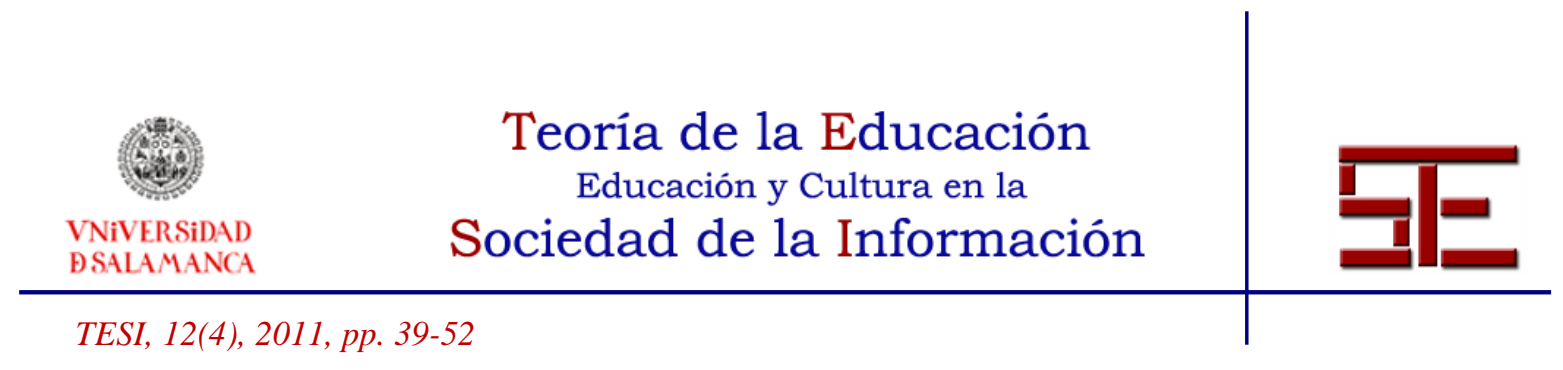

g) INVESTIGA: Se propone la realización de un proyecto en equipo ${ }^{2}$ (los grupos siempre están formados por los niños hermanados, aunque también se pueden completar con otros niños de la misma aula, siempre a criterio del profesor y de acuerdo con la guía didáctica correspondiente) en el que se sintetizan los principales conceptos introducidos en el módulo. Se trata de saber si los alumnos son capaces de aplicarlos a situaciones o contextos concretos, buscando el intercambio de opiniones y experiencias entre niños de los distintos países participantes en e-Culturas. Se busca en todo momento vincular la propuesta a un trabajo colaborativo entre los niños hermanados. Al finalizar el proyecto se debe programar una sesión de videoconferencia para compartir algunos de los trabajos con las aulas hermanas o dentro de las mismas aulas para dar a conocer las diferentes producciones.

h) EVALÚA: Se puede considerar como una evaluación del módulo. Una evaluación cualitativa del trabajo realizado y de la forma en que se ha desarrollado en cada grupo. Adopta la forma de portafolios o Cuaderno virtual, diferente para el alumno y para el profesor.

Esta propuesta metodológica de carácter secuencial, repetida en cada uno de los módulos que componen el Programa Intercultural e-Culturas, se completa con otros tres apartados fijos que estarán presentes en todos los módulos a la vista de los alumnos en el apartado correspondiente de la página web:

- Para saber más: Textos para la ampliación de conocimientos.

- Vocabulario: Palabras en forma de hipertexto que permite una explicación clara y directa del significado de aquellos términos que supongan una dificultad en su comprensión, de manera especial los que hagan referencia a aspectos autóctonos de los países participantes.

- Amplía: Apartado en el que se ofrecen diversas actividades dirigidas a aquel alumnado que termina antes que los demás sus tareas o que desea ampliar los conocimientos sobre el tema. Puede tener diversos grados de dificultad.

\footnotetext{
${ }^{2}$ La fase de Investiga se apoya, según los casos, en la realización de una Webquest sobre el tema tratado.
}

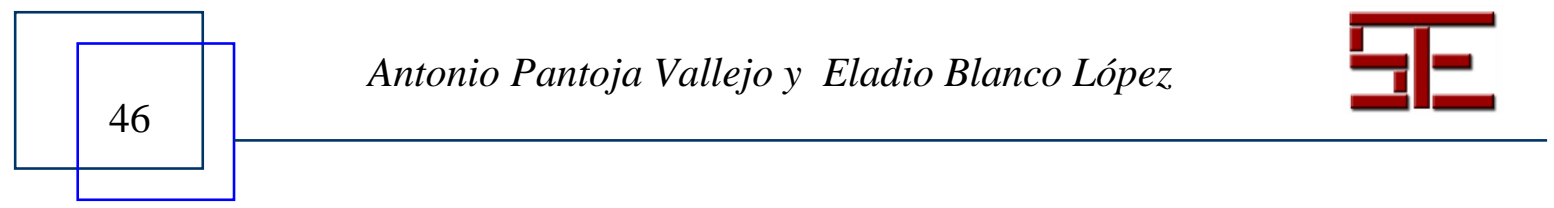




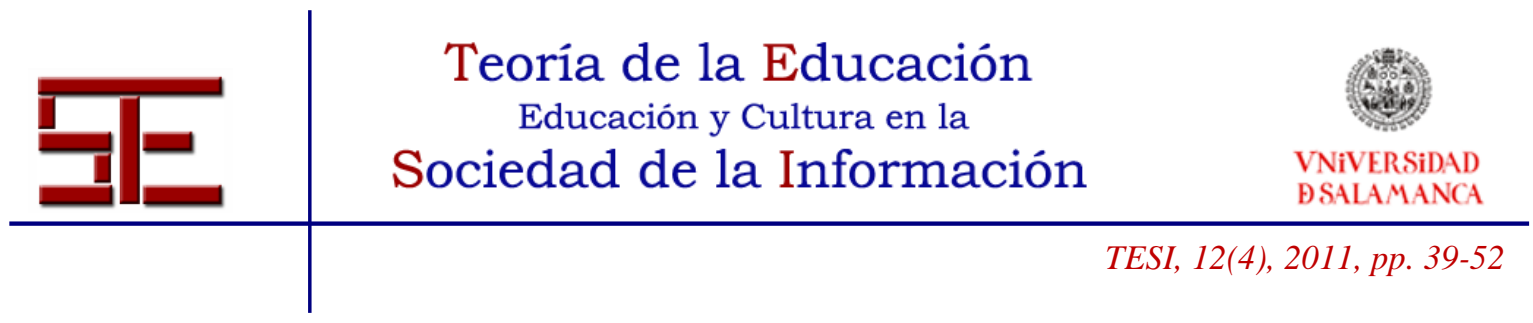

\section{4.- DESCRIPCIÓN DEL SOFTWARE Y ACTIVIDADES UTILIZADAS}

El sistema software de la Red Internacional e-Culturas está compuesto por una plataforma web donde cada usuario, mediante contraseña, tiene acceso a los distintos materiales y recursos, así como noticias, eventos y otra información importante. Dicha información y recursos cambia en función del rol del usuario, esto es, los alumnos tienen una vista de sus actividades, los tutores la tienen de sus distintos alumnos para ver cómo evolucionan y los administradores pueden gestionar diferentes aspectos de la plataforma.

Desde la plataforma web se puede acceder a cada una de las 3 fases del proyecto: Álbum, Mosaico y Quijotín. Cada una de ellas está compuesta por una serie de actividades cuyo fin es el aprendizaje cooperativo de los alumnos hermanados sobre algún tema intercultural. A continuación se describe cada fase junto a alguna de sus actividades más interesantes.

En el Álbum, cada alumno dispone de un espacio donde almacenar fotografías y responder a unas cuestiones que se le piden como si se tratara de un cuaderno personal. Estas fotografías y anotaciones serán visibles sólo por él y sus compañeros hermanados de los diferentes países. Entre las actividades del Álbum se pueden encontrar algunas relacionadas con las fiestas y costumbres de la localidad, sistema educativo del país, gastronomía, deportes, etc. Los alumnos, al rellenar estas actividades con sus fotografías y datos están construyendo un "mural" con la información de los 3 alumnos hermanados, de tal forma que puedan conocer los aspectos anteriores de los países de sus compañeros y, a su vez, compararlos con el suyo propio. A continuación se muestra una imagen de la actividad "Mi Localidad" del Álbum, en la que se tiene que aportar información de la localidad de cada alumno y subir fotografías de la misma, de sus monumentos...

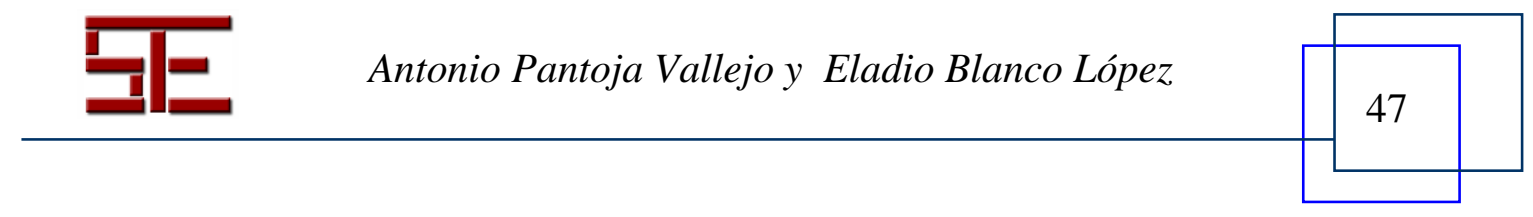




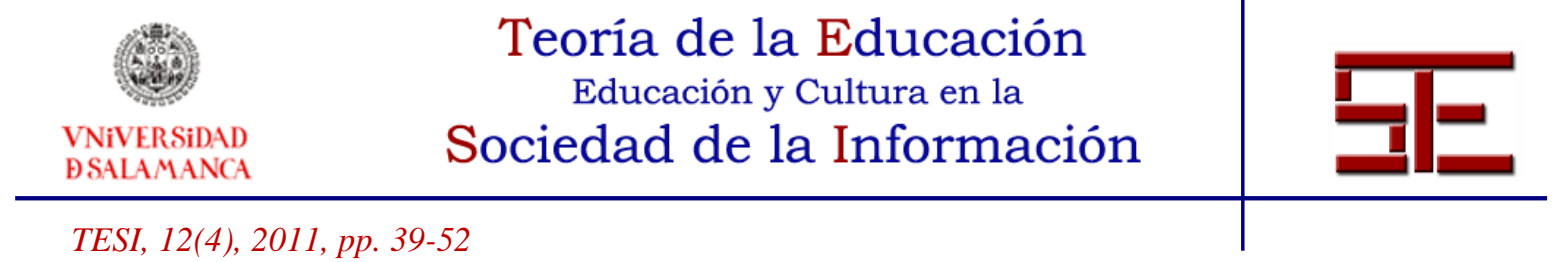

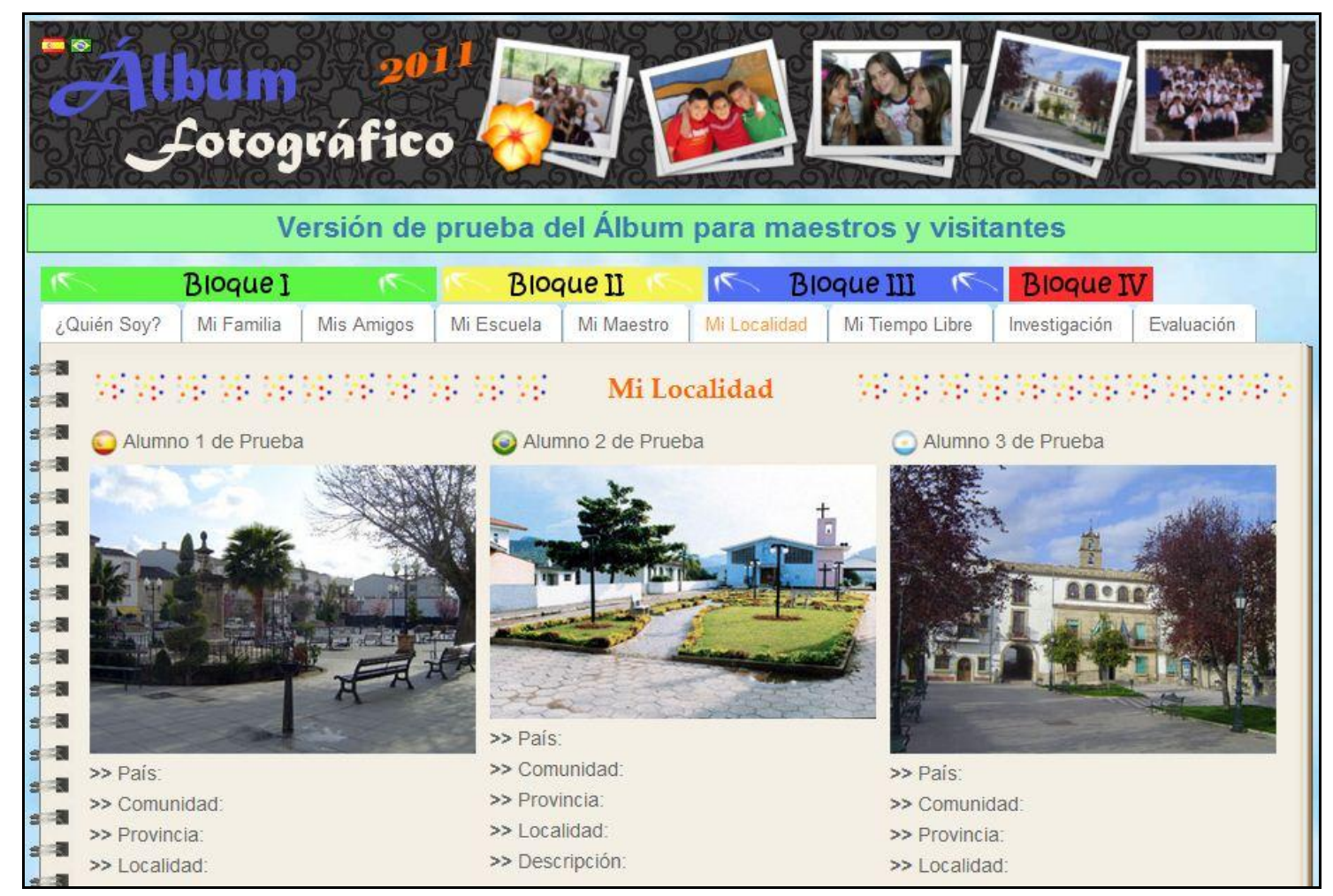

Gráfico 1: Actividad "Mi Localidad" del Álbum.

El Mosaico se compone de multitud de actividades interactivas que tendrán que completar los alumnos por parejas dentro la misma clase. Para resolver muchas de estas actividades necesitarán buscar información en unos recursos proporcionados donde está la respuesta. En otras actividades, como la que se muestra en la siguiente imagen en la que tienen que elaborar una guía turística de su región, tendrán que visitar los recursos proporcionados para conocer lo más representativo del lugar donde viven, obtener fotografías, etc. Una vez elaborada la guía por los alumnos, se realiza un intercambio con la realizada por las demás aulas de los distintos países, para que puedan conocer la misma información que han tenido que rellenar, pero en este caso de los demás países.

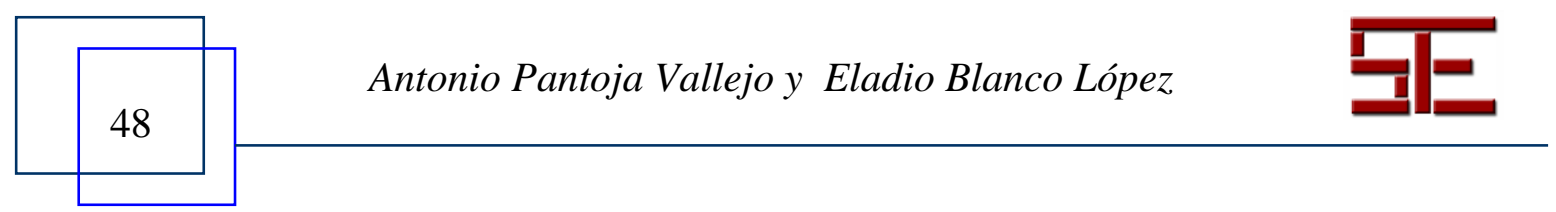




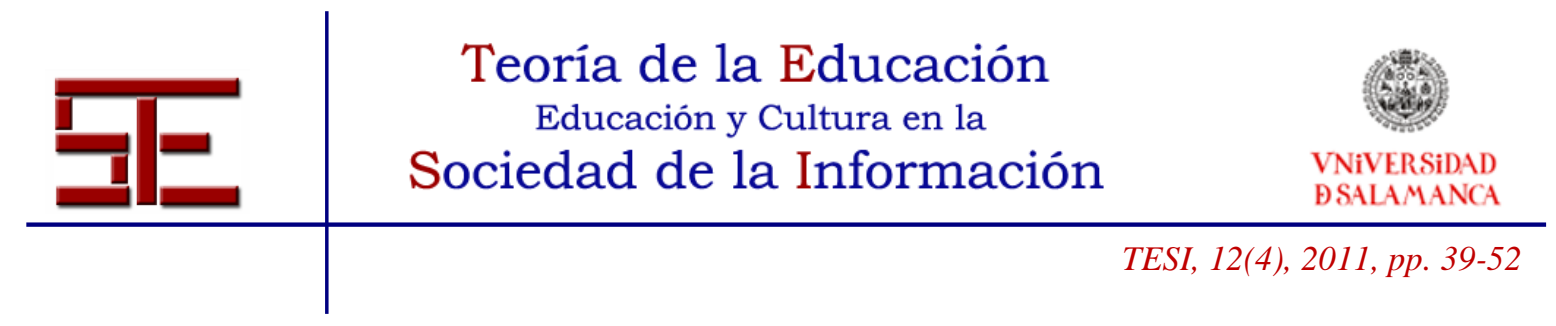

\section{Fase 3: Elabora la "guía turística" \\ Con la información que hayas recogido ya puedes realizar la guía turística, completando los siguientes pasos: \\ - Escribe los nombres de los autores. \\ - Ponle el título al proyecto. \\ - Completa la información que hace falta. \\ - Realiza las actividades interculturales que te proponemos.}

A continuación tienes unos espacios para que completes con la información que has encontrado y que te facilitarán el diseño de la guia. Además también podrás incluir la guía completa que hayáis elaborado.

Escoge un título sugerente y llamativo para la guia turistica, por ejemplo: ¡Así vivimos! ¡La riqueza de un pais!, "Descubre donde vivo..."

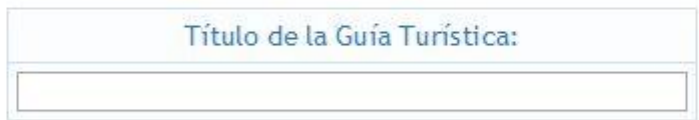

No te olvides de colocar los nombres de los autores que elaboran la guia turística.

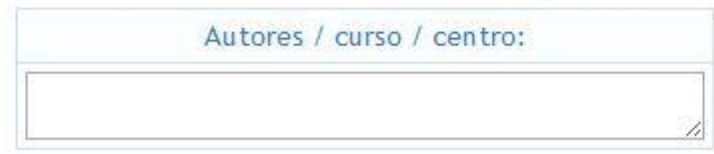

Guardar

Gráfico 2: Actividad "Guía Turística” del Mosaico.

El Quijotín es un juego formado por 6 recorridos interactivos que van desde Jaén hasta Latinoamérica, en los que se van haciendo preguntas al alumno en función de la situación geográfica en la que se encuentre. Son preguntas de respuesta múltiple que, en el caso de que el alumno responda erróneamente, se le dará una nueva oportunidad para contestar, pero en este caso con menor puntuación si acierta. En cada pregunta existe una "Oficina de Información" que proporciona un enlace a un recurso donde se encuentra la respuesta que el alumno tendrá que buscar. Cada alumno del grupo hermanado realiza estos recorridos de forma individual, pero sin poder avanzar al siguiente recorrido mientras no termine todo el grupo el recorrido actual. La puntuación obtenida por todos ellos se suma para obtener la puntuación total del grupo. Esta puntuación se podrá observar en todo momento en un ranking formado por todos los

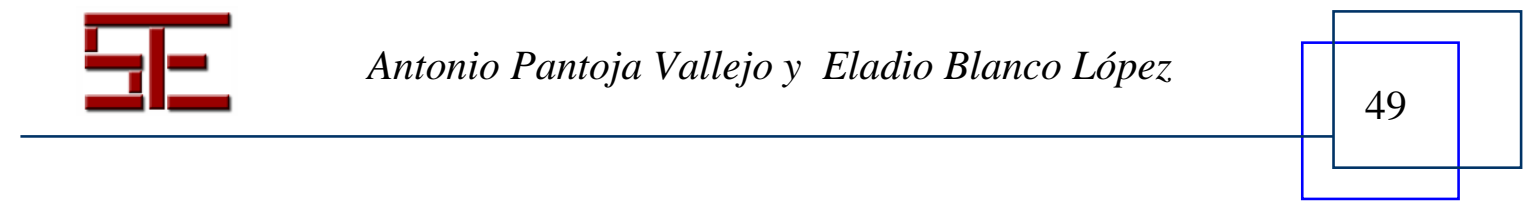




\begin{tabular}{c} 
Teoría de la Educación \\
Educación y Cultura en la \\
$\begin{array}{c}\text { VNiVERSIDAD } \\
\text { BSALAMANCA }\end{array}$ \\
\hline TESI, 12(4), 2011, pp. 39-52
\end{tabular}

grupos para motivar al alumnado a la hora de buscar las respuestas a las preguntas que se le plantean en cada recorrido. Éstas se basan en un conjunto de tópicos interculturales que aparecen junto a ellas: historia, geografía, arte, lengua y literatura, gastronomía, etc. A continuación se muestra una imagen del primer recorrido del Quijotín:

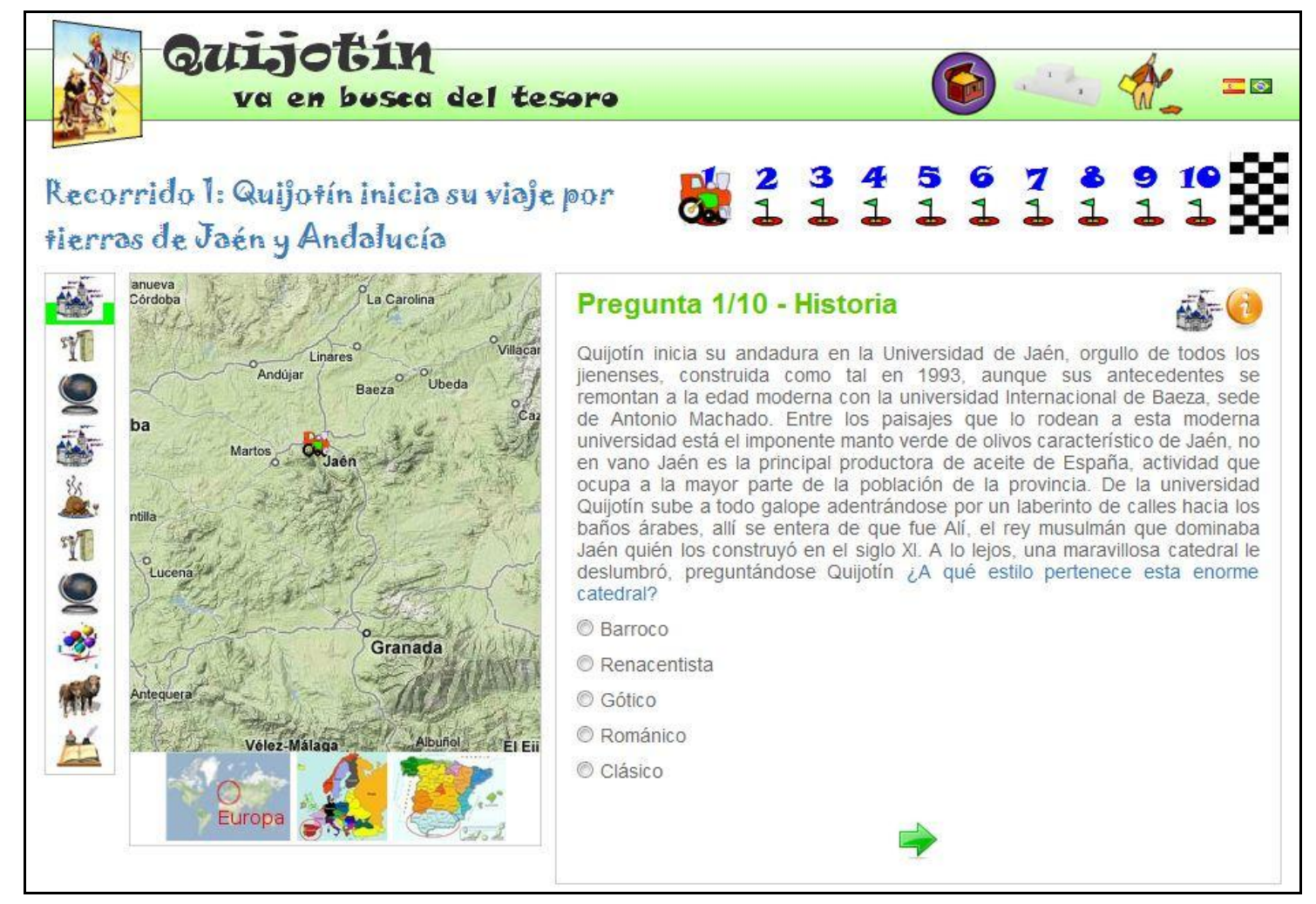

\section{Gráfico 3: El Quijotín.}

Como herramienta transversal a las fases del proyecto, se dispone de un foro para favorecer la comunicación entre todos los integrantes de e-Culturas. Este foro también es usado para promover debates sobre ciertas tareas a lo largo del proyecto y que los alumnos puedan construir una opinión sobre estos aspectos mediante el intercambio con el resto de participantes.

Otra herramienta colaborativa bastante útil cuando se trata de relacionarse con gente de varios países es el "Vocabulario". Mediante él, los alumnos disponen de un listado de palabras con su descripción y términos utilizados en los distintos países, ya que muchas veces para referirse a lo mismo, en lugares distintos se utilizan diferentes palabras. Los

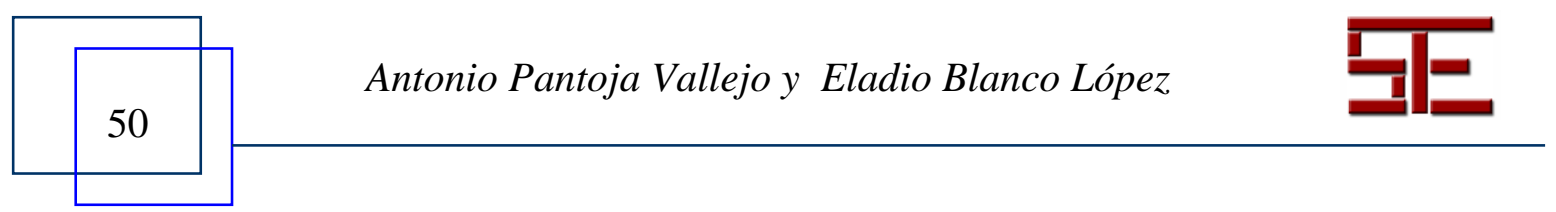




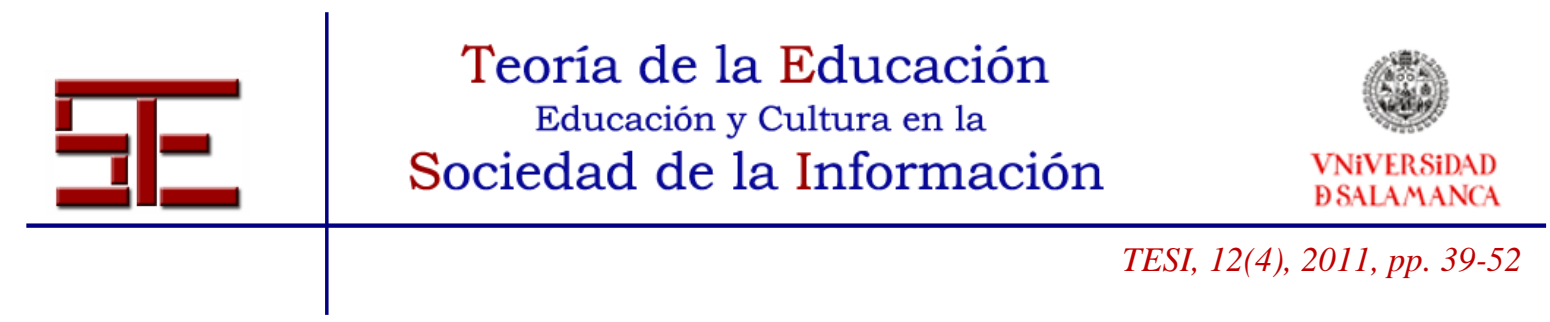

tutores y administradores son los encargados de añadir nuevas palabras y completar los términos o descripciones de las que ya hay. Al final de cada actividad del Álbum, los tutores tienen que añadir las palabras que más hayan llamado la atención a sus alumnos, elaborando así entre todas las aulas el listado de palabras del vocabulario. A continuación se puede observar la vista correspondiente a la palabra "aula" del vocabulario:

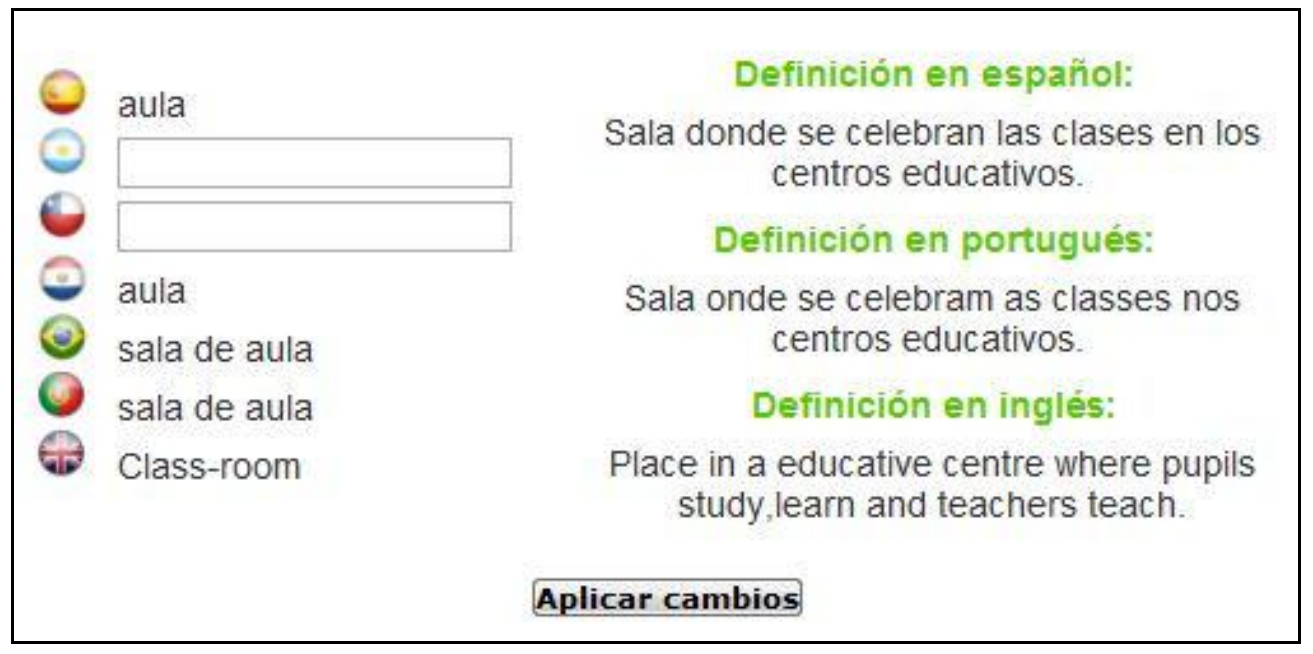

Gráfico 4: Vista de una palabra del vocabulario.

Otros apartados con especial interés son el "Álbum Fotográfico" y "Vídeos" donde se muestran fotografías y vídeos enviados por los tutores para mostrar alguna fiesta o costumbre popular, permitiendo a los alumnos profundizar sobre lo que han conocido de sus compañeros hermanados.

\section{5.- CONCLUSIÓN}

Internet ofrece considerables opciones al trabajo colaborativo, por su capacidad para que sujetos distantes compartan y realicen tareas diversas de manera síncrona o asíncrona. La Red Internacional e-Culturas pone en valor esta posibilidad y explota la capacidad humana de colaborar para construir una sociedad más justa, diversa y solidaria. Esto es sólo el principio de un largo camino que llevará a la constitución de sociedades auténticamente interculturales.

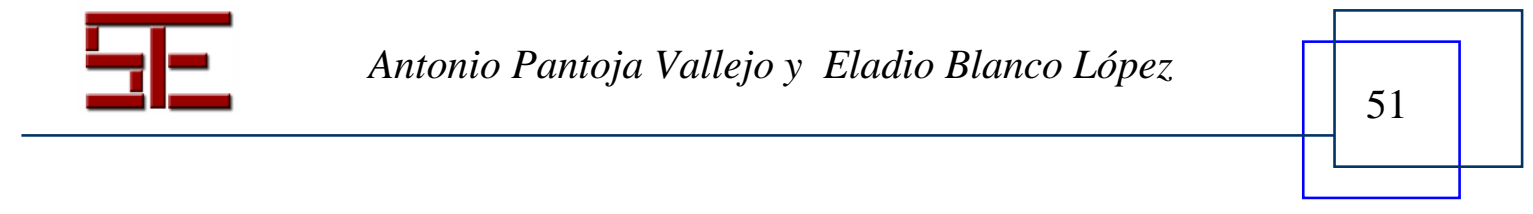




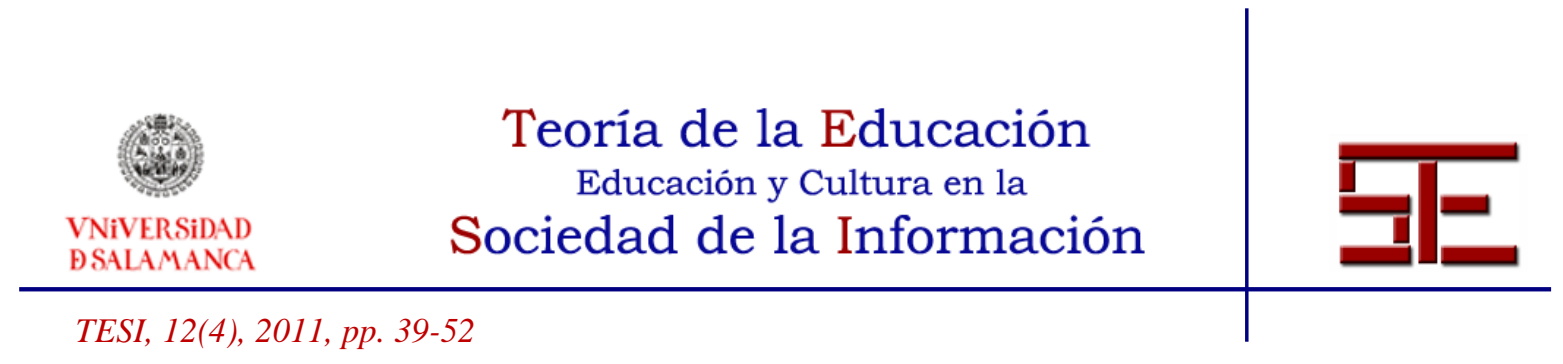

\section{BIBLIOGRAFÍA}

Alcaide, M., Blanco, E., Pantoja, A. y Jiménez, A. S. Capacitación de maestros en valores interculturales a través de la Red Internacional e-Culturas. INECE'08 (Madrid).

Pantoja, A. (1997). Influencia del lenguaje de programación LOGO en la capacidad creativa del niño del Tercer Ciclo de Educación Primaria. Tesis doctoral. Jaén: Servicio de Publicaciones de la Universidad de Jaén.

Pantoja, A. et al. (2006). El proyecto internacional de educación intercultural eCulturas. En T. J. Campoy, A. Pantoja y C. Villanueva (Coords.), V Jornadas sobre Diagnóstico y Orientación. La educación intercultural en la Sociedad de la Información (pp. 1-214). Jaén: Servicio de Publicaciones de la Universidad de Jaén. Edición en CD-Rom.

- (2008). Internet y la construcción de una ciudadanía intercultural. Balance de una experiencia. En A. Pantoja, T. J. Campoy, A. Jiménez y C. Villanueva (Coords.), El carácter universal de la Educación Intercultural. Actas de las I Jornadas Internacionales y VI Jornadas sobre Diagnóstico y Orientación (pp. 72-128). Jaén: Servicio de Publicaciones de la Universidad.

Torre, S. de la (1991). Metodología heurística. En R. Marín y S. de la Torre (Eds.), Manual de la creatividad (pp. 169-189). Barcelona: Vicens-Vives.

Para citar el presente artículo puede utilizar la siguiente referencia:

Pantoja Vallejo, A. y Blanco López, E. (2011). Aprendizaje cooperativo y heurístico en la red internacional e-culturas. Revista Teoría de la Educación: Educación y Cultura en la Sociedad de la Información. 12(4), 39-52 [Fecha de consulta: dd/mm/aaaa]. http://campus.usal.es/ revistas_trabajo/index.php/revistatesi/article/view/8525/8611

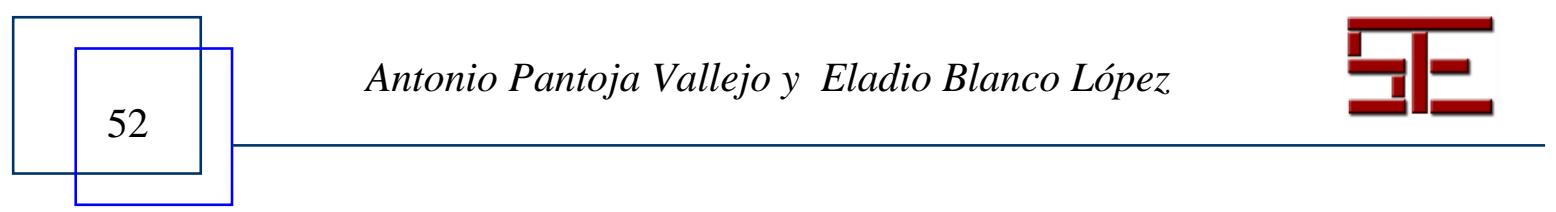

